\title{
Cálculos térmicos \\ y de dinámica de gases del motor GTD350 implementado con una cámara de combustión a volumen constante
}

\section{Gas Dynamics and Thermal Calculations Applied in a Turbo Shaft Engine GTD350, Modified with a Constant Volume Combustion Chamber}

\section{Resumen}

Este artículo está enfocado hacia la determinación de los parámetros de operación del motor GTD 350; este motor opera con cámara de combustión a presión constante utilizando el ciclo Brayton; para la ejecución del estudio térmico se operará con una cámara de combustión a volumen constante la cual emplea el ciclo termodinámico denominado Humphrey.

Mediante este documento se desarrollará el análisis comparativo del comportamiento térmico del motor utilizando el ciclo Brayton (motor base) y operando con el ciclo Humphrey (motor modificado). Se desea comprobar térmicamente que al emplear una cámara de combustión a volumen constante (Ciclo Humphrey) se logrará una disminución en el consumo de combustible, se aumentará la eficiencia y el trabajo desarrollado por el motor base.

\section{Palabras clave}

Rotor de ondas, ciclo Humphrey, eficiencia térmica, entropía, entalpia, flujo de combustible, cámara de combustión.

Ph. D. (c). Universidad Politécnica de Varsovia M. Sc. Ingeniería Aeroespacial con énfasis en Sistemas de Propulsión, Universidad Politécnica de Varsovia. Ingeniero Aeronáutico, Universidad de San Buenaventura, Bogotá. E-mail: rcerpa@usbbog.edu.co

** Ingeniera Aeronáutica (c). Universidad de San Buenaventura, Bogotá. E-mail: jramos@academia.usbbog.edu.co. 


\section{Abstract}

This article is focused on the thermal analysis comparison of the engine GTD 350 operating parameters, using the Brayton and Humphrey cycle The aim of the following research project is to test that applying constant volume combustion chamber in a turbojet engine (Humphrey Cycle) is possible to obtain an increase in the power and thermal efficiency and decrease the fuel consumption in comparison with the baseline engine using Brayton Cycle.

\section{Keywords}

Wave Rotor, Humphrey Cycle, thermal efficiency, constant volume combustion, pulse detonation engines.

\section{Introducción}

Desde los primeros diseños de los sistemas de propulsión se ha buscado el aumento del empuje y del rendimiento térmico de la turbomaquinaria, a lo enunciado anteriormente y de acuerdo a los costos de operación y emisión de contaminantes los estudios se han centrado en la disminución del consumo especifico de combustible (SFC), para ello se han realizado investigaciones en los ciclos aplicados a los motores de turbina, debido a lo enunciado anteriormente se realizará un estudio de la implementación del ciclo Humphrey en un turborreactor, este es un ciclo termodinámico, que combina las mejores características del ciclo Otto (motores de combustión interna) y ciclo Brayton (turbinas de gas); el ciclo Humphrey incluye la combustión a volumen constante (CVC) que garantiza un aumento tanto de la presión como de la temperatura del gas de trabajo como el que se encuentra en un motor de turbina, dando así un resultado de mayor eficiencia térmica ideal, es por esto, que se estudiará la implementación del ciclo Humphrey en un motor turboeje, ya que este elevaría la potencia entregada al eje y el rendimiento térmico , a su vez disminuirá el consumo específico de combustible (SFC) y de esta manera se determinarán los parámetros de operación de la cámara de combustión a volumen constante aplicada al motor GTD350.

En la gráfica 1, se observa cómo el ciclo Humphrey aprovecha las ventajas de: combustión de volumen constante perteneciente al ciclo Otto (1') y la de combustión a presión constante ( $3^{\prime}$ ) como es la del ciclo Brayton, estos dos ciclos termodinámicos al unirse producen un mayor trabajo y a su vez mayor eficiencia con respecto a otros ciclos termodinámicos.

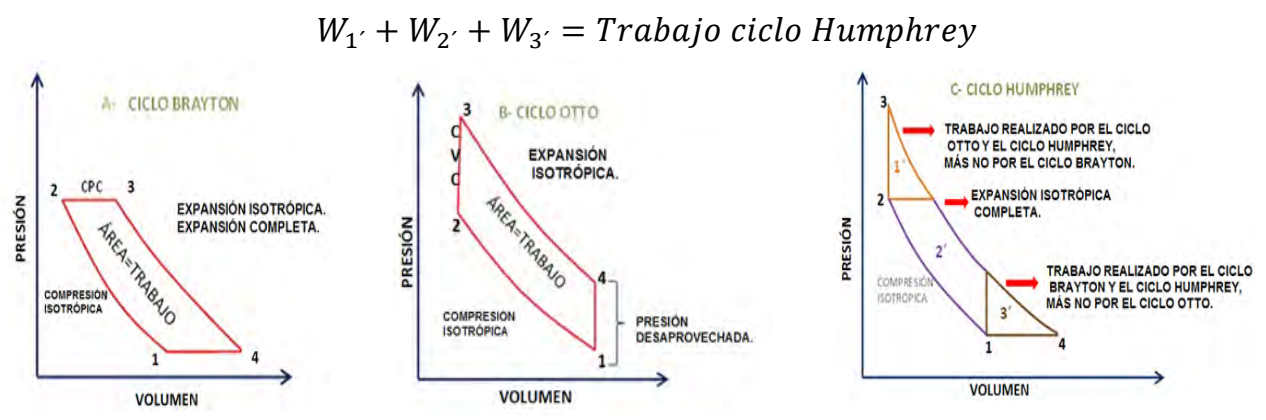

Gráfica 1. Diagrama P-v, ciclos: Brayton, Otto y Humphrey 


\section{Resultados de la investigación}

\section{Cálculos térmicos Turbo eje base.}

Los cálculos térmicos del motor Klimov GTD 35014, se realizarán con base en las condiciones ambiente en Bogotá, los parámetros de entrada para el desarrollo de los cálculos térmicos del motor turboeje base Klimov GTD 350 son:

\begin{tabular}{|l|c|c|}
\hline Parámetro & Valor & Unidades \\
\hline Flujo másico $^{15}$ & 2,1908 & $\mathrm{Kg} / \mathrm{s}$ \\
\hline \hline Relación de Compresión $^{16}$ & $6.05: 1$ & $\mathrm{~K}$ \\
\hline \hline Temperatura de entrada de la turbina $^{17}$ & 1243.15 & Unidades \\
\hline \hline Constante & Valor & $\mathrm{KJ} / \mathrm{KgK}$ \\
\hline $\mathrm{Cp}^{18}$ & 1,005 & $\mathrm{~J} / \mathrm{Kg} \mathrm{K}$ \\
\hline \hline Raire $^{19}$ & 287,1848 & $\mathrm{KJ} / \mathrm{KgK}$ \\
\hline \hline $\mathrm{Cv}^{20}$ & 0,71781 & \\
\hline \hline $\mathrm{K}^{21}$ & 1,4 & \\
\hline
\end{tabular}

Tabla 1. Parámetros iniciales del motor Klimov.

A continuación se muestran las estaciones utilizadas en los cálculos térmicos y de dinámica de gases para el motor base (operando con cámara de combustión a presión constante).

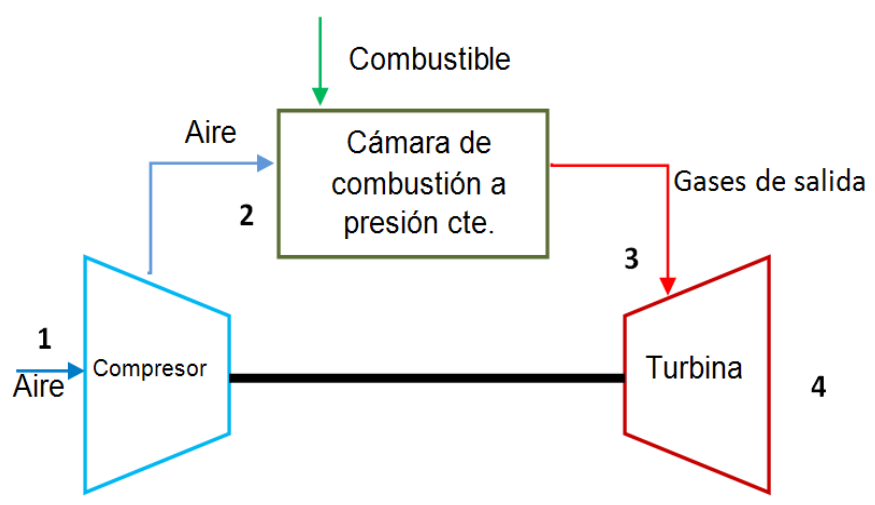

Gráfica 2. Estaciones del motor base «ciclo Brayton».

Los procesos llevados a cabo en un motor turborreactor base son:

1-2 compresión isentrópica.

2-3 Combustión a presión constante.

3-4 Expansión estable e isentrópica

4-1 Entrega de calor al medio ambiente a presión constante.

Los estudios realizados en la investigación, arrojaron los siguientes resultados: 


\begin{tabular}{|c|c|c|c|c|c||}
\hline Estación & Presión (Pa) & Temperatura (K) & Densidad $\mathbf{( K g} / \mathbf{m} 3)$ & Volumen especifico (m3/Kg) & Entropía (J/Kg K) \\
\hline 1 & 101325 & 288,15 & 1,2244 & 0,8167 & 0 \\
\hline \hline 2 & 613016,3 & 481,92 & 4,4292 & 0,2257 & 0 \\
\hline \hline 4 & 613016,3 & 1243,096 & 1,71714 & 0,582364 & 952,2594 \\
\hline \hline 5 & 101325 & 743,2692 & 0,474689 & 2,106643 & 952,2594 \\
\hline
\end{tabular}

Tabla 2. Cálculos térmicos del motor base

\section{Cálculos térmicos turboeje modifícado:}

Estudios realizados por la Universidad Estatal de Michigan (MSU) y la Universidad Politécnica de Varsovia, dieron como resultado, que al implementar una cámara de combustión a volumen constante en los motores de reacción, producen un aumento de presión y temperatura dentro de la cámara de combustión, la adaptación del ciclo Humphrey (combustión de volumen constante), produce mayor entalpía en el turboeje modificado y por tanto mayor trabajo producido en el ciclo. Para el fácil entendimiento del análisis térmico del motor modificado, se muestran a continuación las estaciones de este.

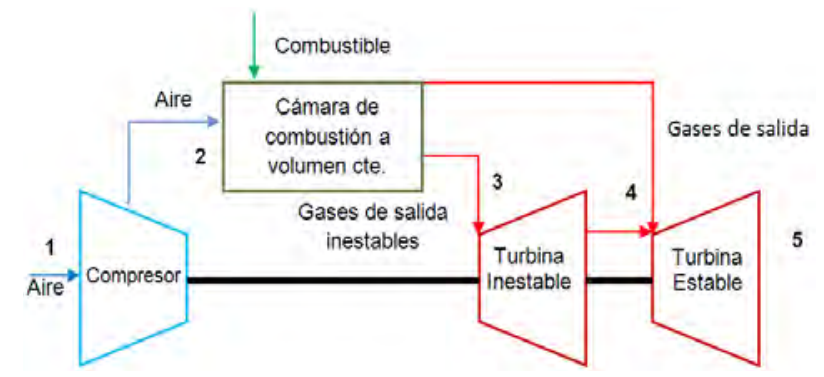

Gráfica 3. Estaciones del motor modificado «ciclo Humphrey»

En un motor turborreactor modificado con el ciclo Humphrey se realizan los siguientes procesos:

1-2 compresión isentrópica.

2-3 Combustión a volumen constante.

3-4 Expansión del fluido inestable.

4-5 Expansión isotrópica del fluido estable.

5-1 Entrega de calor al medio ambiente a presión constante.

Para el motor modificado con ciclo Humphrey, los parámetros obtenidos en los cálculos térmicos son mostrados en la tabla 3:

\begin{tabular}{|c|c|c|c|c|c|}
\hline Estación & Presión (Pa) & Temperatura (K) & Densidad (Kg/m3) & Volumen especifico (m3/Kg) & Entropía (J/Kg K) \\
\hline 1 & 101325 & 288,15 & 1,2244 & 0,8167 & 0 \\
\hline 2 & 613016,3 & 481,92 & 4,4292 & 0,2257 & 0 \\
\hline 3 & 2309992 & 1816 & 4,4292 & 0,2257 & 952,2594 \\
\hline 4 & 613016,3 & 1243,09 & 1,7171 & 0,5823 & 952,2594 \\
\hline \hline 5 & 101325 & 743,26 & 0,4746 & 2,1066 & 952,2594 \\
\hline
\end{tabular}

Tabla 3. Cálculos térmicos del motor modificado. 
Teniendo en cuenta las tablas del motor base y del motor modificado se pueden obtener los diagramas temperatura-entropía ( $T$-s) presión-volumen (P-V).

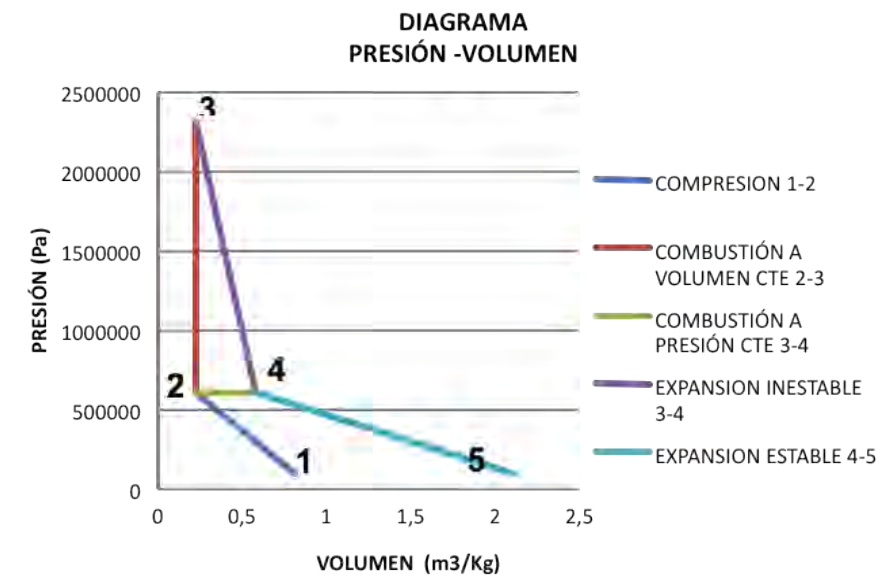

Gráfica 4. Presión vs. Volumen específico para el motor modificado

En el diagrama P-v, se observa la comparación del ciclo Brayton (1-2-4-5) y el ciclo Humphrey (1-2-3-4-5); en el cual de 1-2 se produce una compresión isotrópica en el compresor; 2-3 Combustión de volumen constante en la cámara de combustión. Esta combustión emplea el concepto del ciclo Humphrey, este proceso comparado con el del punto 2-4 combustión a presión constante, genera en el ciclo termodinámico un trabajo adicional que produce el aumento de la potencia, dando como resultado una enorme ventaja con respecto al ciclo utilizado hasta el momento en los turborreactores; finalmente de 3-4 se produce una expansión inestable y de 4-5 el gas realiza una expansión estable en la turbina.

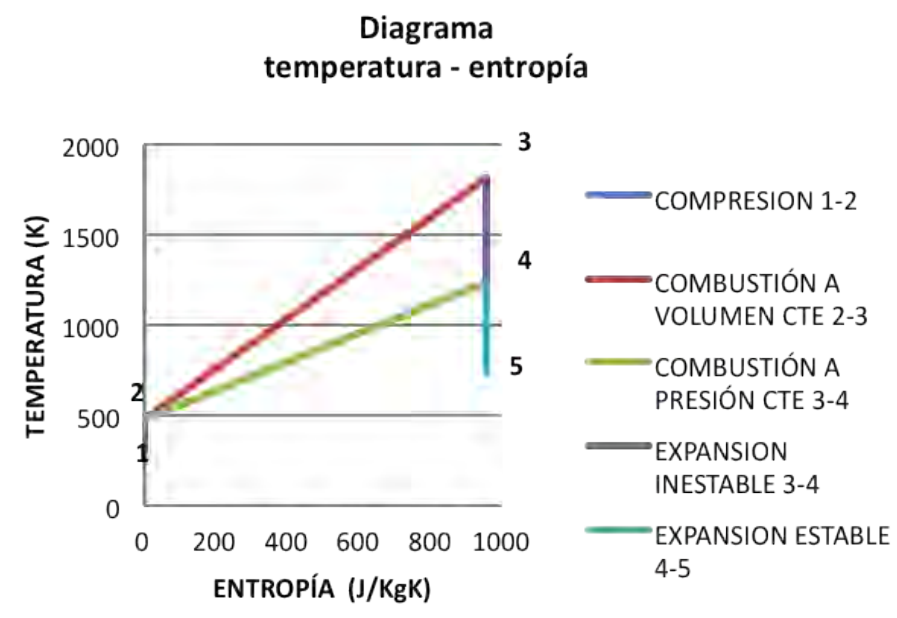

Gráfica 5. Temperatura vs. entropía para el motor modificado.

En la gráfica T-s nuevamente se observa la comparación del ciclo Brayton (1-2-4-5) y el ciclo Humphrey (1-2-3-4-5); de 1-2 tiene un proceso de compresión isentrópico, generando un aumento de presión y temperatura; de 2-3 Combustión de volumen constante (ciclo Humphrey) y combustión de presión constante 2-4. (Ciclo Brayton). Se puede concluir que el ciclo que trabaja la combustión a volumen constante tendrá un mayor incremento de energía (Temperatura y presión) y esta energía podrá ser convertida también en tra- 
bajo; aumentando la potencia producida por el motor. Ahora bien en el punto 3-4 y 4-5 se puede ver que hay una expansión inestable y estable respectivamente, al igual que en el diagrama presión-volumen.

En la gráfica 6 se puede observar que el trabajo realizado por el motor modificado (ciclo Humphrey) genera un incremento de 62,63\%, en comparación con el motor base. Con lo cual se puede comprobar la teoría que al implementar en un motor turborreactor una cámara de combustión a volumen constante, es posible incrementar el trabajo producido por el mismo.

Gráfica comparativa trabajo producido en cada ciclo

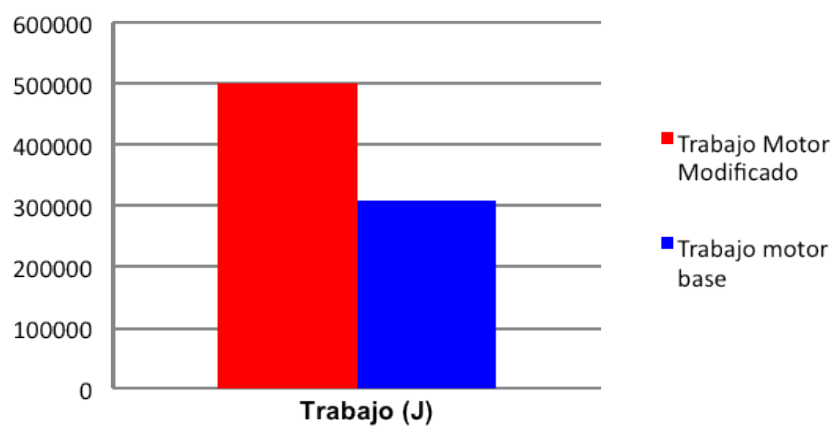

Gráfica 6. Trabajo realizado para cada motor

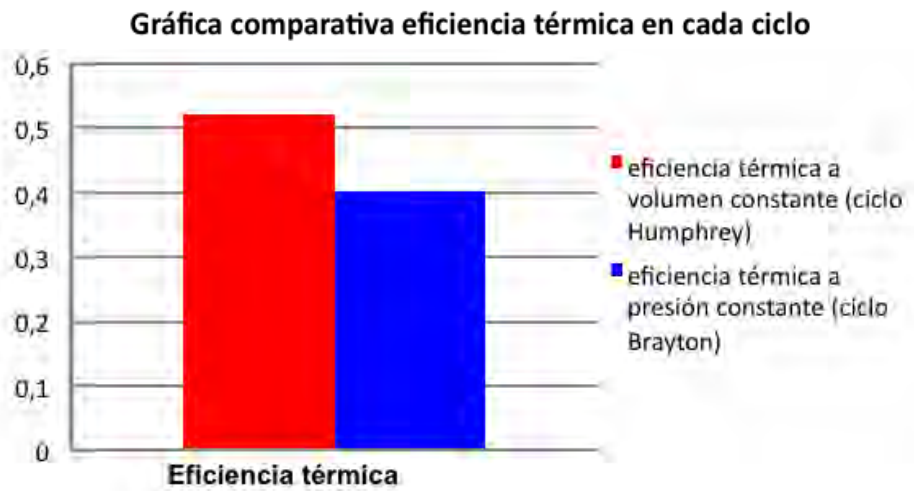

Gráfica 7. Comparación de eficiencia térmica de cada ciclo termodinámico

Gráfica comparativa consumo de combustible

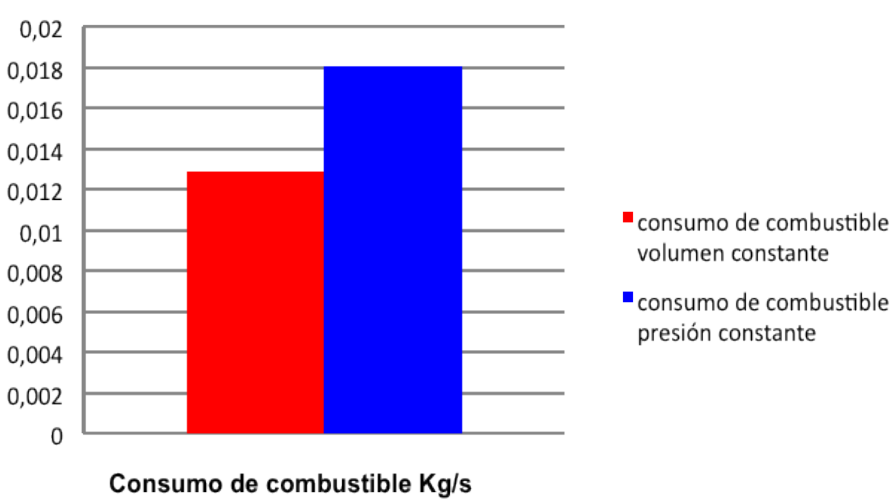

Gráfica 8. Comparación de consumo de combustible de cada ciclo 
Según la gráfica 8, efectivamente se observa que el motor que tiene implementada la cámara de combustión de volumen constante tiene una reducción en el consumo de combustible de $40 \%$, por lo tanto se demuestra la teoría explicada en la introducción del documento y que en la actualidad es la ventaja más importante de la implementación del ciclo Humphrey. Ya que si se tienen en que dichos resultados el motor base produciría menor cantidad de $\mathrm{CO}_{2}$ y disminuiría los costos de operación por combustible de la aeronave.

Finalmente se puede resumir que implementando una cámara de combustión a volumen constante en un motor turborreactor (Ciclo Humphrey), el trabajo del motor se incrementa en un $62.63 \%$, de igual manera ocurre con la eficiencia térmica ya que aumenta en un $29,91 \%$ y el consumo de combustible disminuirá en un $40 \%$. Comprobando a través de los cálculos térmicos y de dinámica de gases la teoría expuesta sobre la ventaja del uso del ciclo Humphrey en los aerorreactores.

\section{Conclusiones}

- Se comprobó a través de los cálculos térmicos y de dinámica de gases la teoría expuesta sobre la ventaja del uso del ciclo Humphrey, que enuncia que al implementar una cámara de combustión de volumen constante, se produce mayor trabajo, mayor rendimiento térmico, y menor consumo de combustible respecto al motor base.

- Modificando el motor Klimov GTD 350 con una cámara de combustión de volumen constante, se obtiene un aumento del $62,63 \%$ en el trabajo producido por el motor, $29,91 \%$ en la eficiencia térmica y una disminución del $40 \%$ en el consumo de combustible.

- Al producir menos consumo de combustible se puede generar menor cantidad de emisión de gases efecto invernadero como lo es el $\mathrm{CO}_{2}$, el cual depende de la cantidad de combustible (hidrocarburo) quemado en el proceso de combustión.

- Implementando un ciclo Humprey en un aerorreactor se pueden suprimir etapas de compresión, debido a que en el proceso de combustión se genera un abrupto incremento de la presión y temperatura del fluido de trabajo del motor.

\section{Referencias}

[1] Akbari, Pejman, Müler, N. «Wave Rotor Research Program at Michigan State University.AlAA» Propulsion Conference and Exhibit, n. ${ }^{\circ} 41$. July 2005.

[2] Dempsey, Emmett, Müller, Norbert. «The Ultra-micro Wave Rotor Research at Michigan State University. The 2nd International Symposium on Innovative Aerial/Space Flyer Systems». Diciembre 2005, The University of Tokyo, p. 11.

[3] Escobar, Arnold, Cerpa, Rafael, Piechna, J. y Rico, María Camila. "Cálculos términos y de dinámica de gases de un turbojet "powergeneration x-01", modificado con un rotor de ondas". revista Ingenium, 2011 pp. 56-65.

[4] Fatsis, A., Vrachopoulos, G., Mavrommatis, S., Panoutsopoulou \& A y Layrenti, F. A computational method for pressure wave machinery to internal combustion engines and gas turbine. (13 de febrero de 2013).

[5] Kailasanath, K., «Review of Propulsion Applications of Detonation Waves», AIAA Journal, Vol. 39, n.․ 9, 2000, pp. $1698-1708$.

[6] Mayer. A computational method for pressure wave machinery to internal combustion engines and gas turbine, 2009 , p. 8.

[7] Okamoto, K. and Nagashima, T., «A Simple Numerical Approach of Micro Wave Rotor Gasdynamic Design». ISABE, 20031213, 2003. 
[8] Piechna, J. «Wave Machines Models and Numerical Simulation». En:_OficinaWydawniczaPolitechniki Warszawskiej, 2005.

[9] Piechna, Janusz and Dyntar, David. "Numerical investigation of the Wave Disk Micro-Engine concept». International Journal of Gas Turbine, Propulsion and Power Systems. Diciembre, Vol. 2, n. ${ }^{\circ}$ 1, 2008.

[10] PIECHNA, Janusz. Wave Machines, Models and Numerical Simulation. Varsovia. Oficyna Wydawnicza Politechniki Warszawskiej, 2005.

[11] Piechna, J., Akbari, P., Lancu, F. and Müller, N., «Radial-Flow Wave Rotor Concepts, Unconventional Designs and Applications», IMECE 2004-59022, ASME, Anaheim, CA, 2004.

[12] Cerpa, R., Piechna, J., Muller, N. «Numerical Analysis of the Untypical Effects in a Wave Topping Unit for a Small Turbojet», Revista de la Facultad de Ingeniería, n. ${ }^{\circ} 20,2009$.

[13] Piechna, J., Escobar, A, Müller, N. "Project of a Small Turbo Jet Engine Topped by the Wave Machine», The $3^{\text {rd }}$ International Symposium on Innovative Aerial/Space Flyer Systems The University of Tokyo, nov. 2006, 24-25,

[14] Official web Klimov. Principal specification. Our products R \& D [en linea], 2004-2012. [citado agosto de 2012]. Disponible en internet http://en.klimov.ru/production/helicopter/GTD-350/

[15] Ishikawajima-Harima Heavy Industries, en: Flight International. 2 de junio de 1969, p. 23.

[16] Parámetros de operación motor GTD-350, disponible en; http://en.klimov.ru/production/helicopter/GTD-350/

[17] Gunston, Bill. World Encyclopedia of Aero Engines. Cambridge, England. Patrick Stephens Limited, 1989.

[18] Granger, Robert. Experiments in heat transfer and thermodynamics. University of Cambridge. (C) Cambridge University Press. 1994.

[19] Potter, Merler, C. y Wiggert, David. Mecánica de fluidos. 3. a edición, Thompson, 2002.

[20] Yunus, Cengel y Boles, Michael. Termodinámica. 6. edición, Mexico: McGraw-Hill, 2008. ISBN 978-0-07-352921-9.

[21] Heiser, W. H. and Pratt D. T. «Thermodynamic Cycle Analysis of Pulse Detonation Engines», Journal of Propulsion and Power, Vol. 18, n. 1, January-February 2002.

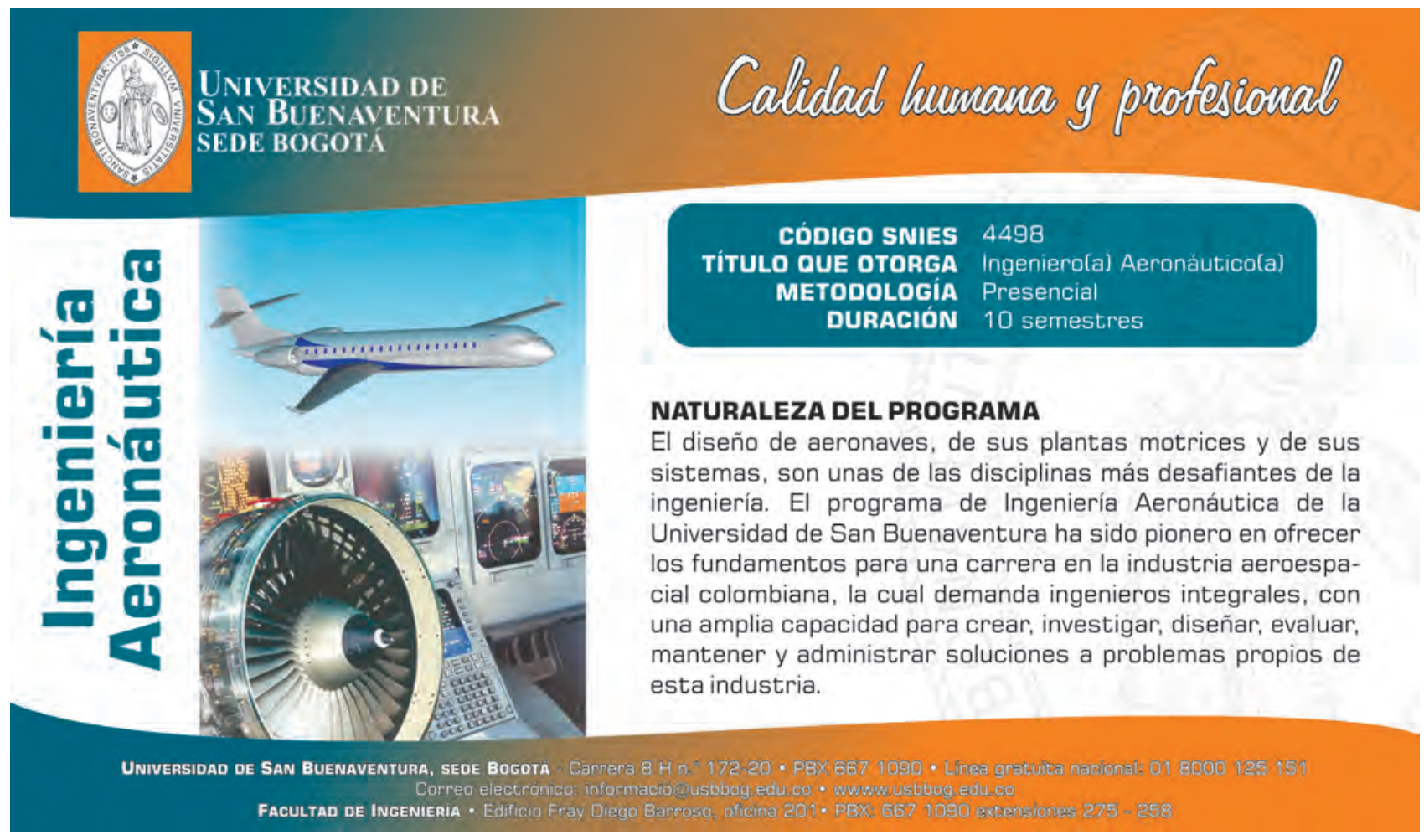

